\title{
Gene therapies should be for all
}

\author{
Are healthcare systems prepared to deal with cost and accessibility of gene therapies on a global scale?
}

M ore than 200 phase 2 and 3 gene therapy trials are currently underway, which could translate into up to 40 new products' being approved for clinical use in the next decade, and the potential eligibility of 1.09 million patients for this therapeutic modality in the next 15 years. The availability of more gene therapy products will bring profound changes to the treatment landscape of many rare genetic diseases, which will offer for the first time potentially curative options for patients. Healthcare systems worldwide have to start preparing now to cope with the challenge of ensuring that all patients, not just a select few with financial means and privileged access to technology, can benefit from these innovative therapies.

Cost is at the center of the gene therapy accessibility problem. In many countries, drug prices are regulated and subject to existing laws; however, for gene therapies, pricing remains largely unregulated and is decided on a case-by-case basis, often concentrating on a single upfront payment. When determining the price, drug companies consider development costs, the specific characteristics of the disease and the related expenses, including hospitalizations and missed productivity, that can potentially be prevented by decreasing the disease burden. In the case of gene therapies, prices are also affected by lack of competition, with a limited number of companies developing gene therapies for rare diseases, and by the fact that often only a small number of patients can benefit from a specific treatment, a situation in which profits from a small number of doses need to offset the entire drug-development and manufacturing costs.

The first gene therapy product to be approved for a congenital disorder in the United States was voretigene neparvovec, for the treatment of vision loss due to inherited retinal dystrophy; this was launched in the market in 2017 at a cost of US $\$ 425,000$ per eye for a single dose. More recently, onasemnogene abeparvovec, for the treatment of pediatric patients with spinal muscular dystrophy, became the most expensive drug on the market, costing US\$2.1 million per dose. With the handful of gene therapies already on the market costing on average 30 times the median household income in the United States per dose, there are growing concerns that not all patients will be able to afford these treatments, and this will exacerbate existing inequalities.

The worry is that the commercialization of gene therapies might further penalize disadvantaged communities and ethnic groups that, on average, have lower incomes and more restricted access to healthcare. This year, the American Society for Gene and Cell Therapy set leveling the playing field and improving equitable access to gene therapies worldwide as a top priority. The World Health Organization has also called for the development of global standards for human genome editing, highlighting the need for an equity agenda to ensure that everyone can access the benefits of this technology.

Sickle cell disease-a genetic blood condition that affects predominantly people of African ancestries-is a good case study. Approximately 100,000 people in the United States live with this disease, and half are enrolled in Medicaid programs. As therapeutic options are limited and a single mutation in a well-studied gene causes the condition, sickle cell disease is considered an ideal candidate for gene therapy, and nine clinical trials are currently underway. Experts believe that gene therapy products for sickle cell disease will probably cost US $\$ 1$ million for a one-time dose. A recent report estimated that offering a lentivirus-based therapy to eligible Medicaid enrollees with severe sickle cell disease could result in serious affordability problems. Concerns have been raised that Medicaid programs may decide to implement strategies to limit access to the potentially life-saving therapy to only a limited number of patients. Innovative payment models are therefore urgently needed to ensure equitable access to these therapies.

Proposed alternatives include outcomes-based pricing - whereby the price is proportional to the benefit that individual patients get from the therapy-staggered payments to mitigate the high upfront costs, and even 'healthcoin', a proposed currency that represents health outcomes and can be converted into marketplace currencies. Multiple calls have also been made to ensure the price tag reflects any taxpayer money used during the drug-development process.

In countries in which the healthcare system is centralized, as in many European nations, access to innovative therapies is also far from homogeneous. Individual countries agree on prices with drug companies independently, often under secret negotiations, after the European Medicine Agency grants approval. For example, in 2019, conditional approval by the this agency was granted to betibeglogene autotemcel, a one-time gene therapy for beta thalassemia. While discussions with different European countries are ongoing, the drug manufacturer suspended negotiations with payers in Germany last month after failing to reach a deal. Calls have also been made for an improvement in cross-border healthcare legal provisions to prevent the scenario in which patients with the same condition cannot access life-saving therapies that are available for free in a neighboring country.

Potentially one-off curative gene therapies for diseases such as sickle cell disease hold particular promise for patients in low- and middle-income countries, where the long-term management of the disease is particularly challenging due to resource-strained healthcare systems. Limited research capacity and a paucity of technologically advanced facilities have also prevented many low- and middle-income countries from participating in the development and deployment of gene therapies.

International collaborations could help to bridge this gap. The Bill \& Melinda Gates Foundation, in partnership with the US National Institutes of Health and Novartis, has launched programs aimed at identifying relevant partners in sub-Saharan African countries for collaborative international gene therapy basic and clinical research over the next decade. Efforts are also ongoing to develop accessible in vivo gene therapy options that would enable circumventing the need to modify patients' cells ex vivo and would thus provide a more feasible option in the Global South.

Although it is unlikely that a unified, global solution will overcome all of the accessibility challenges, moving forward, drug companies, payers, policymakers and patient-advocacy groups need to work together to develop innovative solutions to ensure that access to gene therapies is truly equitable.

Published online: 12 August 2021 https://doi.org/10.1038/s41591-021-01481-9 